\title{
Effect of tree-level and mean-field improvement on the light-hadron spectrum in quenched QCD
}

\author{
H. P. Shanahan ${ }^{*}$ and C. T. H. Davies \\ Department of Physics \& Astronomy, University of Glasgow, Glasgow, G12 8QQ, Scotland, United Kingdom \\ K. C. Bowler, R. D. Kenway, D. G. Richards, P. A. Rowland, and S. M. Ryan \\ Department of Physics \& Astronomy, The University of Edinburgh, Edinburgh EH9 3JZ, Scotland, United Kingdom \\ P. Lacock and C. Michael \\ Theoretical Physics Division, Department of Mathematical Sciences, University of Liverpool, Liverpool L69 3BX, United Kingdom \\ D. R. Burford and N. Stella \\ Department of Physics, University of Southampton, Southampton SO17 1BJ, United Kingdom \\ H. Wittig \\ DESY-IfH, Zeuthen, Platanenalle 6, D-15738 Zeuthen, Germany
}

(UKQCD Collaboration)

(Received 14 August 1996)

\begin{abstract}
We compute the light-hadron mass spectrum at $\beta=5.7$ using the $O(a)$-improved Sheikholeslami-Wohlert fermion action with two choices of the clover coefficient: the classical value $c=1$ and a mean-field or tadpole-improved estimate $c=1.57$. We compare our results with those of the GF11 Collaboration who use the Wilson fermion action $(c=0)$. We find that changing $c$ from zero to 1 and 1.57 leads to significant differences in the masses of the chirally extrapolated and strange pseudoscalar and vector mesons, the nucleon, the $\Delta$, and also in the Edinburgh plot. A number of other quantities, for example, $m_{V}^{2}-m_{P}^{2}, J, a m_{K} / a m_{\rho}$, and $a m_{K^{*}} / a m_{\rho}$ do not appear to change significantly. We also investigate the effect of changing the lattice volume from approximately $(2 \mathrm{fm})^{3}$ to $(2.6 \mathrm{fm})^{3}$. We find that the meson masses are consistent to within one standard deviation and baryon masses are consistent to within two standard deviations.
\end{abstract}

[S0556-2821(97)04803-0]

PACS number(s): 12.38.Gc, 11.15.Ha, 14.65.Bt

\section{INTRODUCTION}

The ab initio calculation of the light-hadron spectrum is a major goal of lattice QCD. A calculation of the light-hadron spectrum giving results in good agreement with experiment would be a demonstration that QCD describes long-distance strong-interaction physics. Furthermore, the calculation is an essential precursor to the calculation of other nonperturbative observables in QCD, such as $B_{K}, B_{B}$, leptonic, and semileptonic decay matrix elements and the moments of the nucleon structure function. Lattice calculations are, however, subject to systematic errors from the nonzero lattice spacing, the finite volume of the lattice, the extrapolation in the valence quark mass to the chiral limit, and the quenched approximation. In this paper, the effects of the first two sources of error will be examined.

Symanzik [1] proposed an improvement program for reducing the dependence of observables on the lattice spacing, $a$, by adding to the action higher-dimension operators with appropriately calculated coefficients. This should enable a more reliable extrapolation to the continuum limit, using data at larger values of the lattice spacing. Given that the computational effort scales as $a^{-6}$ in the quenched approximation, the potential savings are considerable.

The standard gluon action has discretization errors of

\footnotetext{
*Electronic address: H.Shanahan@physics.gla.ac.uk
}

$O\left(a^{2}\right)$. The Wilson fermion action, on the other hand, has discretization errors of $O(a)$. Therefore, the first step in the Symanzik improvement program is to reduce the leadingorder error of the fermion action to the same order as that of the gluon action. The resulting Sheikholeslami-Wohlert (SW) action [2] introduces an extra operator, $P(x)$, the socalled clover term, to the original action, multiplied by a parameter $c$ :

$$
S_{\mathrm{SW}}^{F}=S_{W}^{F}(\kappa, r)+a^{4} c \kappa r \sum_{x} \bar{\psi}_{x} P(x) \psi_{x}
$$

where $S_{W}^{F}(\kappa, r)$ is the standard Wilson action defined as

$$
\begin{aligned}
S_{W}^{F}(\kappa, r)= & a^{4} \sum_{x}\left\{\bar{\psi}_{x} \psi_{x}+\kappa \sum_{\mu}\left[\bar{\psi}_{x}\left(\gamma_{\mu}-r\right) U_{\mu}(x) \psi_{x+\hat{\mu}}\right.\right. \\
& \left.\left.-\bar{\psi}_{x+\hat{\mu}}\left(\gamma_{\mu}+r\right) U^{\dagger}(x) \psi_{x}\right]\right\},
\end{aligned}
$$

and

$$
P(x)=\frac{-i a}{2} \sum_{\mu, \nu} F_{\mu \nu}^{c}(x) \sigma_{\mu \nu},
$$

$F_{\mu \nu}^{c}(x)$ is a lattice definition of the field strength tensor, detailed in [3]. 
There is a value of the parameter $c, c_{\text {nonpert }}$, which removes all $O(a)$ errors from spectral quantities [4,5]. In this paper, we compare the spectrum obtained using the Wilson fermion action $(c=0)$ with that obtained using the SW fermion action with two choices of $c$ : the classical value, $c=1$, and a mean-field or tadpole-improved estimate of $c_{\text {nonpert }}$. Other approaches to improvement are described in Refs. [6-10].

The tadpole-improved estimate of $c$ is obtained following Lepage and Mackenzie [11] by replacing the gauge links, $U_{\mu}(x)$ by

$$
\widetilde{U}_{\mu}(x)=\frac{1}{u_{0}} U_{\mu}(x)
$$

We choose

$$
u_{0}=\left\langle\frac{1}{3} \operatorname{Tr} U_{\square}\right\rangle^{1 / 4} .
$$

Consequently, the effect of tadpole improvement on the SW action is to set

$$
\begin{gathered}
c=\frac{\widetilde{c}}{u_{0}^{3}}, \\
\kappa=\frac{\widetilde{\kappa}}{u_{0}} .
\end{gathered}
$$

Tree-level theory should then provide more reliable estimates of $\widetilde{c}$ and the critical value of $\widetilde{\kappa}$ which we denote $\widetilde{\kappa}_{\text {crit }}$; we take $\widetilde{c}=1$ and expect $\widetilde{\kappa}_{\text {crit }}$ to be close to $1 / 8$. This prescription maintains the $O(a)$ improvement and it is believed that the size of the remaining discretization error will be reduced.

The paper is organized as follows. In the next section we outline the computational methods. In Sec. III, we explore three values of the clover coefficient at $\beta=5.7$ by including the results from the GF11 Collaboration [12]. The observables studied are: the $\rho$ and $\pi$ masses, vector pseudoscalar mass splittings, the $J$ parameter (proposed by Lacock and Michael [13]), valence $\bar{s}$ meson masses, the spin $1 / 2$ and $3 / 2$ baryon masses, and the Edinburgh plot. A study is also made of possible finite size effects by computing the spectrum at a smaller lattice volume, using one value of the clover coefficient. Finally, in Sec. IV, we present our conclusions.

\section{COMPUTATIONAL DETAILS}

\section{A. Simulation parameters}

Two lattice sizes, $12^{3} \times 24$ and $16^{3} \times 32$, at $\beta=5$.7, were used, with 482 configurations generated on the former and 142 configurations on the latter. We used a combination of the over-relaxation (OR) algorithm [14] and the CabbiboMarinari (CM) algorithm [15]. The gauge configurations were separated by 100 compound sweeps, where a compound sweep is defined as five OR sweeps followed by one CM sweep. A detailed description of the algorithms used can be found in [3].

Quark propagators were calculated at two $\kappa$ values. These values were chosen so that the corresponding quark masses
TABLE I. Hadron operators. The quark fields may be smeared and there is an implicit sum over spatial sites. Lower case latin variables indicate color indices.

\begin{tabular}{ccccc}
\hline \hline & State & $J^{P}$ & Correlators & $\Gamma$ structure \\
\hline Mesons & $\mathrm{P}$ & $0^{-}$ & $\left\langle P(t) P^{\dagger}(0)\right\rangle$ & $P=\bar{\psi}^{a} \gamma_{5} \psi^{a}$ \\
& $V$ & $1^{-}$ & $\left\langle\vec{V}_{1}(t) \cdot \vec{V}_{1}^{\dagger}(0)\right\rangle$ & $\vec{V}_{1}=\overline{\psi^{a}} \vec{\gamma} \psi^{a}$ \\
& & & $\left\langle\vec{V}_{2}(t) \cdot \vec{V}_{2}^{\dagger}(0)\right\rangle$ & $\vec{V}_{2}=\overline{\psi^{a}} \vec{\gamma} \gamma_{4} \psi^{a}$ \\
Baryons & $N$ & $\frac{1}{2}^{-}$ & $\left\langle N_{1}(t) \bar{N}_{1}(0)\right\rangle$ & $N_{1}=\varepsilon_{a b c}\left(\psi^{a} C \gamma_{5} \psi^{b}\right) \psi^{c}$ \\
& & & $\left\langle N_{2}(t) \bar{N}_{2}(0)\right\rangle$ & $N_{2}=\varepsilon_{a b c}\left(\psi^{a} C \gamma_{4} \gamma_{5} \psi^{b}\right) \psi^{c}$ \\
& $\Delta$ & $\frac{3}{2}^{-}$ & $\langle\Delta(t) \bar{\Delta}(0)\rangle$, & $\Delta=\varepsilon_{a b c}\left(\psi^{a} C \gamma_{\mu} \psi^{b}\right) \psi^{c}$ \\
\hline \hline
\end{tabular}

straddle the strange quark mass. On the larger lattice, propagators were calculated using both $c=1$ and the tadpoleimproved value of $c=1.57$. On the smaller lattice, propagators were calculated using the tadpole-improved value of $c$ only.

To increase the overlap of the operators with the ground state, all of the propagators were calculated using both a local source and a Jacobi-smeared source with r.m.s. radius of $2.2 a$ [16]. Local sinks were used for all propagators. The propagators were calculated using the minimal residual algorithm, which is described in detail in [3].

The correlators used to extract the hadron masses are listed in Table I; for further details see [17]. We computed meson correlators using quarks degenerate and nondegenerate in mass, giving three possible mass combinations for each meson state. Furthermore, each quark propagator can be either local or smeared, giving three possible correlators for each mass combination. However, we computed baryon correlators only for degenerate quark masses, using either all smeared or all local quark propagators. Therefore, for each baryon state we have two mass combinations each with two types of sources. In order to maximize the sample size, the discrete time symmetry of the correlators was utilized and the data for $t \in[0, T / 2]$ averaged with the data at $T-t$, where $T$ is the temporal size of the lattice.

These calculations were performed on the Meiko i860 Computing Surfaces at the Edinburgh Parallel Computing Centre.

\section{B. Fitting}

We have performed multiexponential fits of meson correlators to

$$
\sum_{\vec{x}}\left\langle 0\left|M(\vec{x}, t) M^{\dagger}(0)\right| 0\right\rangle=\sum_{n=0}^{n_{\max }} A_{n} \cosh \left(m_{n}\left(\frac{T}{2}-t\right)\right),
$$

and baryon correlators to

$$
\begin{aligned}
\sum_{\vec{x}}\langle 0|B(\vec{x}, t) \bar{B}(0)| 0\rangle= & \sum_{n=0}^{n_{\max }}\left[B_{n} \exp \left(-m_{n} t\right)\right. \\
& \left.+C_{n} \exp \left(-m_{n}^{P}(T-t)\right)\right] .
\end{aligned}
$$



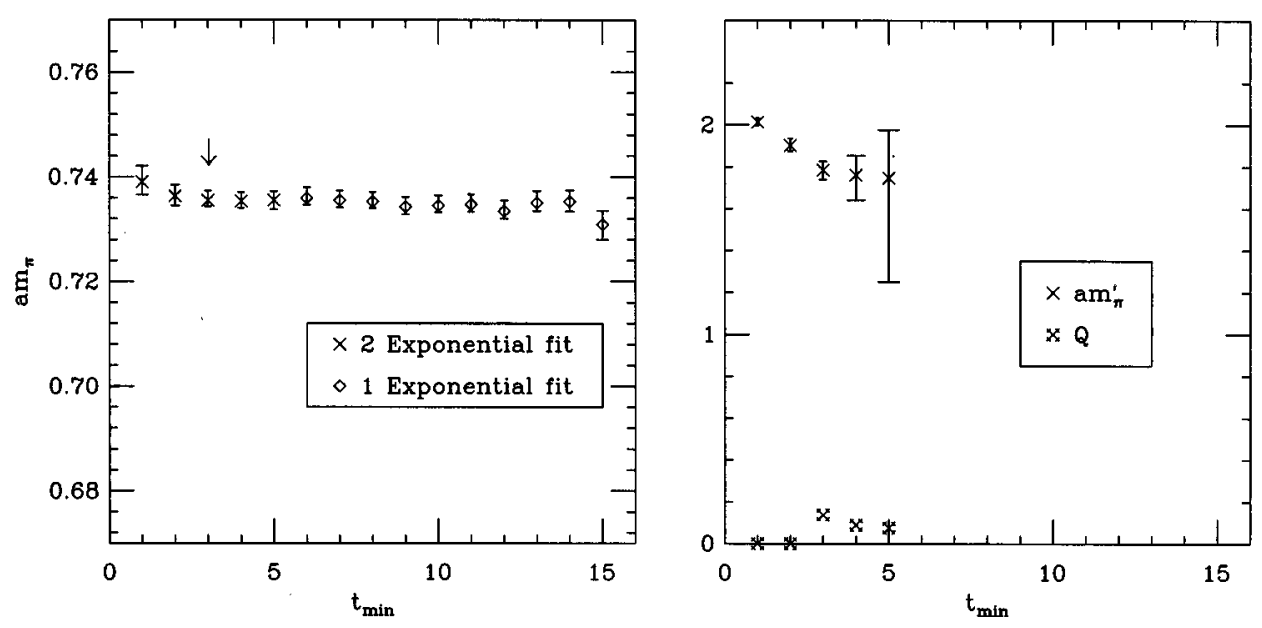

FIG. 1. $a m_{P}, a m_{P}^{\prime}$, and $Q$ versus $t_{\min }$ for $c=1.57,16^{3} \times 32$, $\kappa_{1}, \kappa_{2}=0.13843$, using local and smeared propagators. The arrow indicates which fit range was used for the final result.
$B_{n}$ is the amplitude of the state labeled by $n$, and $C_{n}$ is that of the (heavier) parity partner and $n_{\max } \geqslant 1$.

The following criteria for multiexponential fits have been used: acceptable values for the quality of fit, $Q$, and $\chi^{2} / N_{\mathrm{DF}}$; stability of the result for the ground state mass; agreement between the result obtained using a singleexponential fit and a double-exponential fit; ability of the fitting algorithm to resolve two masses.

The variable $Q$, which is a function of $\chi^{2}$ and $\nu=N_{\mathrm{DF}}$ is defined [18] as

$$
Q\left(\nu, \chi^{2}\right) \equiv \frac{1}{\Gamma(\nu / 2)} \int_{\chi^{2} / 2}^{\infty} e^{-t} t^{\nu / 2-1} d t
$$

It represents the probability that given $\nu$ normal, random, uncorrelated variables, with a mean of 0 and unit variance, have a sum of squares which is greater than $\chi^{2}$. An acceptable value for $Q$ lies around 0.5 ; a much smaller value indicates that the model used is incorrect, whereas a value approaching 1 indicates that too many parameters are being used. A criterion of stability which we used is that the mass obtained does not change noticeably when the minimum time slice of the fit was changed slightly. The parameters were determined by minimizing the $\chi^{2}$ using the LevenbergMarquardt algorithm $[18,19]$. Correlations between all time slices, and types of operator for simultaneous fits, were in- cluded. The covariance matrix was inverted using singular value decomposition, without eliminating any eigenvalues. The bootstrap algorithm [20], using 1000 bootstrap subsamples, was used to determine the $68 \%$ confidence levels, regenerating the covariance matrix for each subsample.

Examples of the multiexponential fits for the pseudoscalar, vector, nucleon, and $\Delta$ are shown in Fig. 1 to Fig. 4 . We emphasize that these are not effective mass plots, but plots of the mass obtained for a given fixed $t_{\max }$ and varying $t_{\min }$. In obtaining results for the smaller lattice, despite having significantly larger statistics, it was more difficult to satisfy the above fit criteria than for the larger lattice. The pseudoscalar mass was determined using all available smearing types and a 2-exponential fit. Fit ranges of 3-12 and 3-16 were chosen for the smaller and larger volumes, respectively. In the case of the vector, the high statistics at the smaller volume allowed the use of both $\Gamma$ matrices, listed in Table I, while for the larger lattice, only $\vec{V}_{1}=\bar{\psi} \vec{\gamma} \psi$ was used. All three different smearing types were used in both fits. Fit ranges of 4-12 and 4-16 were used and a 2-exponential fit.

As can be seen in Fig. 5 there is significant second and even third state contamination for the nucleon when local and smeared operators are used in the fit. Hence, only those correlators calculated with smeared operators, with overlap onto the $J^{P}=1 / 2^{-}$state, were used to determine $a m_{N}$. The contribution of the parity partner of Eq. (9) was found to be
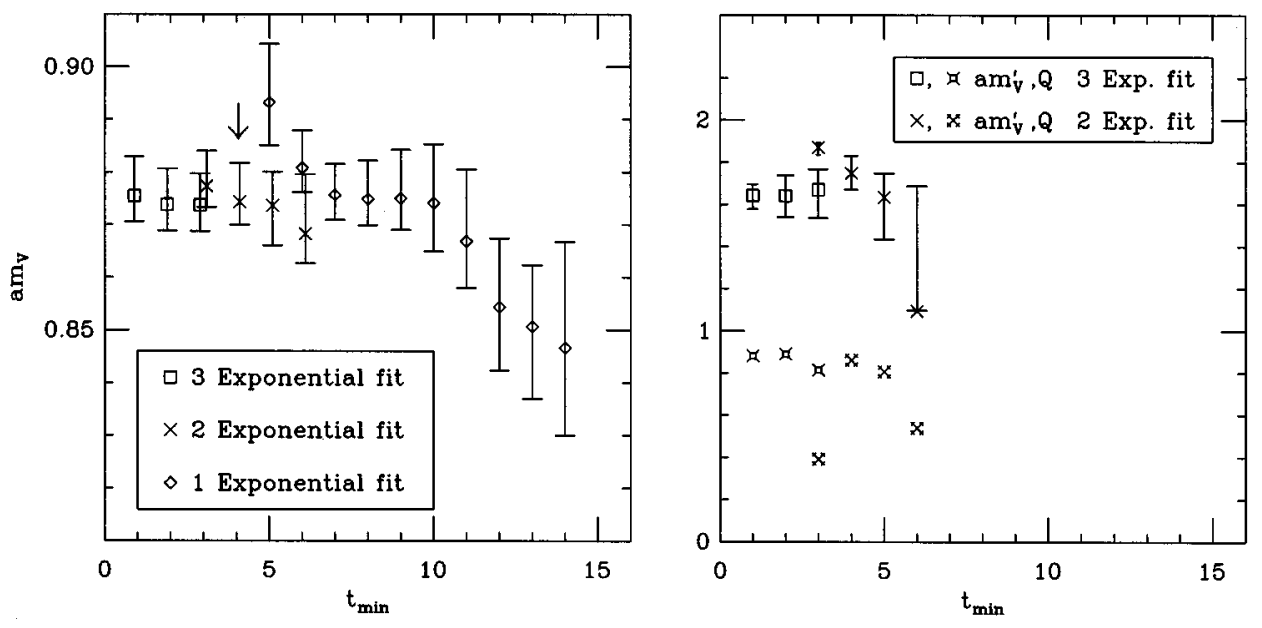

FIG. 2. $a m_{V}, a m_{V}^{\prime}$, and $Q$ versus $t_{\min }$ for $c=1.57$ at $16^{3} \times 32, \quad \kappa_{1}=0.14077, \quad \kappa_{2}$ $=0.13843$ using local and smeared propagators. The arrow indicates which fit range was used for the final result. 

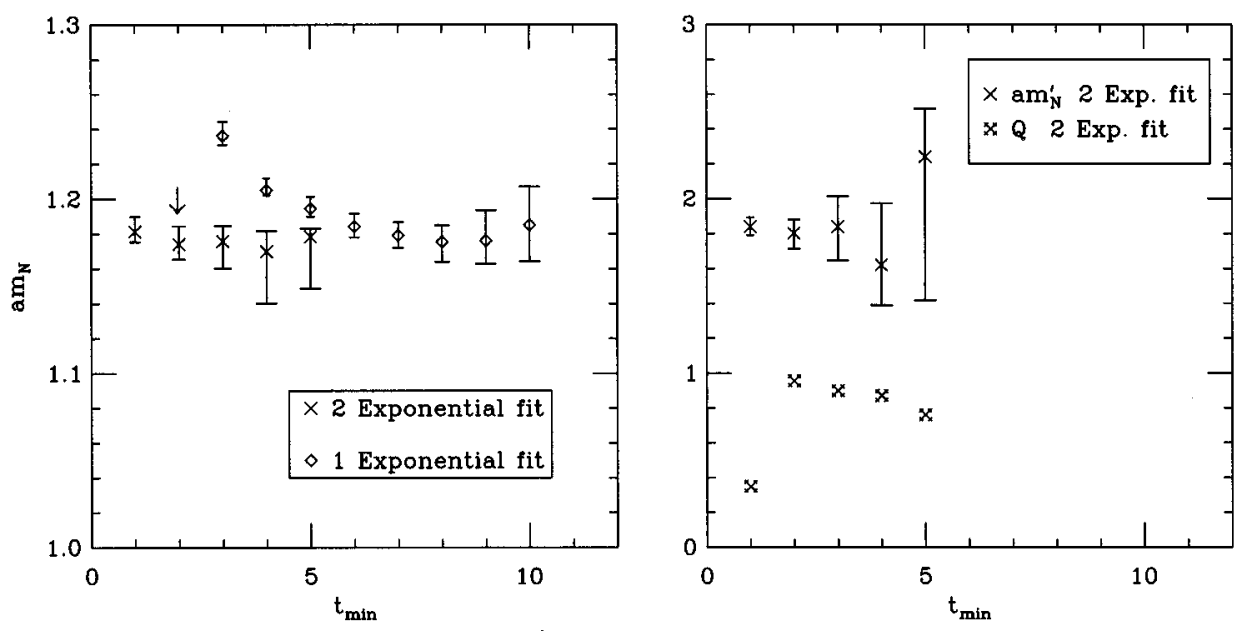

FIG. 3. $a m_{N}, a m_{N}^{\prime}$, and $Q$ versus $t_{\min }$ for $c=1.57,12^{3} \times 24$, $\kappa=0.14077$, using only smeared propagators. The arrow indicates which fit range was used for the final result.
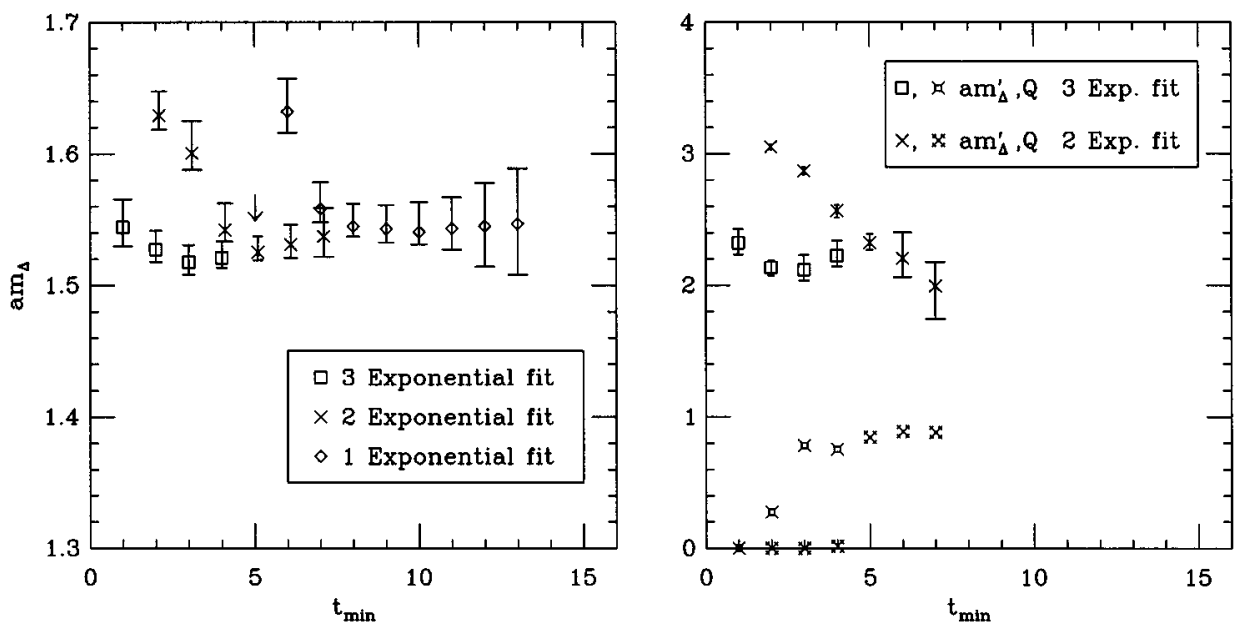

FIG. 4. $a m_{\Delta}, a m_{\Delta}^{\prime}$, and $Q$ versus $t_{\min }$ for $c=1.57,16^{3} \times 32$, $\kappa=0.13843$, using local and smeared propagators. The arrow indicates which fit range was used for the final result.
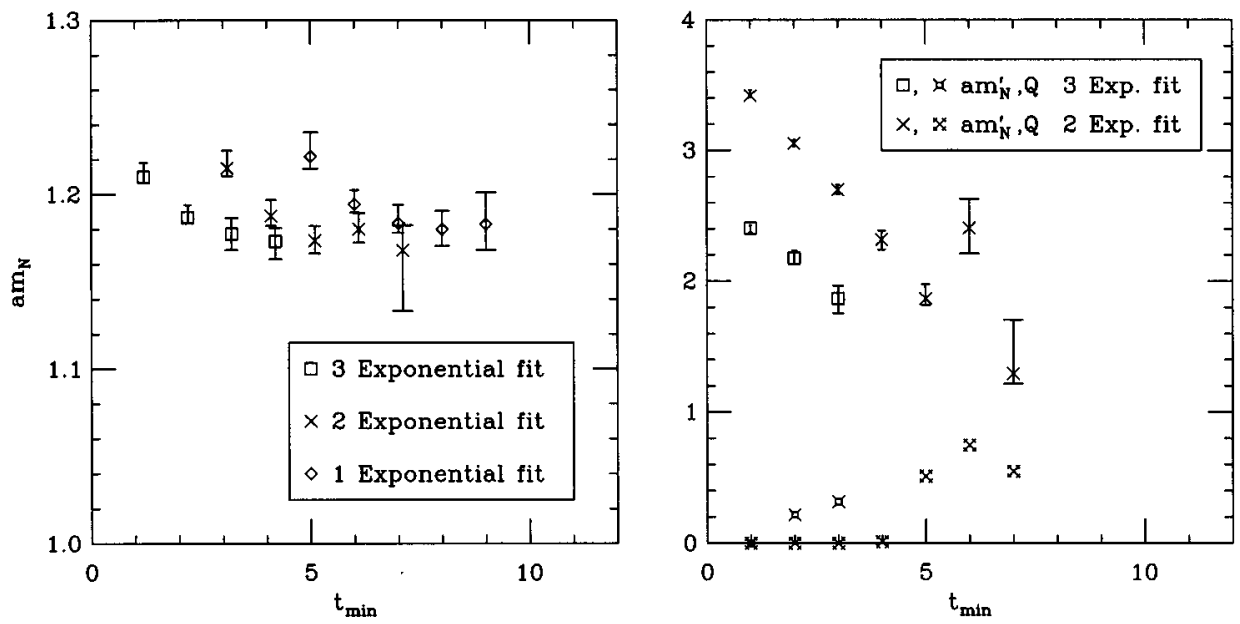

FIG. 5. $a m_{N}, a m_{N}^{\prime}$, and $Q$ versus $t_{\min }$ for $c=1.57,12^{3} \times 24$, $\kappa=0.14077$, using local and smeared propagators. 
TABLE II. Pseudoscalar masses at both volumes and values of $c$.

\begin{tabular}{ccccccccc}
\hline \hline$c$ & $N_{s}^{3} \times N_{t}$ & $\kappa_{1}$ & $\kappa_{2}$ & $a m_{P}$ & Fit range & $\chi^{2}$ & $N_{\text {DF }}$ & $Q$ \\
\hline 1.57 & $12^{3} \times 24$ & 0.13843 & 0.13843 & $0.7361_{-14}^{+18}$ & $3-12$ & 14.8 & 22 & 0.872 \\
1.57 & $12^{3} \times 24$ & 0.14077 & 0.13843 & $0.6384_{-8}^{+18}$ & $3-12$ & 15.6 & 22 & 0.834 \\
1.57 & $12^{3} \times 24$ & 0.14077 & 0.14077 & $0.5292_{-8}^{+21}$ & $3-12$ & 17.7 & 22 & 0.724 \\
& & & & & & & & \\
1.0 & $16^{3} \times 32$ & 0.14663 & 0.14663 & $0.7343_{-13}^{+19}$ & $3-16$ & 33.9 & 34 & 0.474 \\
1.0 & $16^{3} \times 32$ & 0.14948 & 0.14663 & $0.6458_{-12}^{+22}$ & $3-16$ & 37.1 & 34 & 0.326 \\
1.0 & $16^{3} \times 32$ & 0.14948 & 0.14948 & $0.5462_{-12}^{+25}$ & $3-16$ & 43.8 & 34 & 0.121 \\
& & & & & & & & \\
1.57 & $16^{3} \times 32$ & 0.13843 & 0.13843 & $0.7355_{-12}^{+18}$ & $3-16$ & 43.0 & 34 & 0.139 \\
1.57 & $16^{3} \times 32$ & 0.14077 & 0.13843 & $0.6402_{-12}^{+22}$ & $3-16$ & 44.8 & 34 & 0.102 \\
1.57 & $16^{3} \times 32$ & 0.14077 & 0.14077 & $0.5319_{-11}^{+27}$ & $3-16$ & 53.6 & 34 & 0.017 \\
\hline \hline
\end{tabular}

sufficiently suppressed if $t_{\max }$ was chosen to be $T-1$. The fit ranges, using a 2-exponential fit, were 2-11 and 2-15.

In the case of the $\Delta$, the higher state contamination was not as large as for the nucleon. Therefore, local and smeared operators were used. The fit ranges were 5-11 and 5-15 with a 2-exponential fit.

\section{RESULTS}

The masses obtained for the pseudoscalar, vector, nucleon, and $\Delta$ for each value of the clover coefficient and combination of quark masses, are listed in Table II to Table $\mathrm{V}$. The larger lattice size corresponds to one used by the GF11 collaboration with the Wilson fermion action and the same $\beta[12]$, so that we are also able to compare results for nonzero $c$ with those for $c=0$. One expects the effect of changing $c$ will be more noticeable at our coarse lattice spacing than at a larger $\beta$. The effect of reducing the physical volume to $12^{3} \times 24$ was also investigated, using the tadpoleimproved SW action.

\section{A. Effect of clover coefficient}

\section{The chiral limit}

For small quark masses, the bare mass of a quark on the lattice can be defined as

$$
a m_{q}=\frac{1}{2}\left(\frac{1}{\kappa}-\frac{1}{\kappa_{\mathrm{crit}}}\right),
$$

where $\kappa_{\text {crit }}$ is a priori an undetermined function of $\beta$. We use the standard extrapolation in quark mass for pseudoscalar mesons, neglecting possible logarithmic divergences described by Sharpe [21]:

$$
\left(a m_{P}\right)^{2}=b_{\kappa}+\frac{c_{\kappa}}{\kappa}+O\left(\kappa^{-2}\right)
$$

where

$$
\kappa_{\mathrm{crit}}=-\frac{c_{\kappa}}{b_{\kappa}} .
$$

However, as noted by Bhattacharya et al. [22] and Collins et al. [23], the terms which are $O\left(\kappa^{-2}\right)$ cannot be entirely neglected for the quark masses used in this study. A linear extrapolation in $1 / \kappa$ leads to a large $\chi^{2} / N_{\mathrm{DF}}$, as can be seen in Table VI. An estimate of the systematic uncertainty was obtained by performing a quadratic fit through the three masses and a linear fit to the two lightest masses. In all the cases considered, the deviation from the original linear fit was greater for the quadratic fit than for the linear fit to the two lightest masses. The systematic error quoted in Table VI

TABLE III. Vector masses at both volumes and values of $c$.

\begin{tabular}{ccccccccc}
\hline \hline$c$ & $N_{s}^{3} \times N_{t}$ & $\kappa_{1}$ & $\kappa_{2}$ & $a m_{V}$ & Fit range & $\chi^{2}$ & $N_{\text {DF }}$ & $Q$ \\
\hline 1.57 & $12^{3} \times 24$ & 0.13843 & 0.13843 & $0.9381_{-20}^{+33}$ & $4-12$ & 46.2 & 40 & 0.230 \\
1.57 & $12^{3} \times 24$ & 0.14077 & 0.13843 & $0.8775_{-26}^{+39}$ & $4-12$ & 39.6 & 40 & 0.488 \\
1.57 & $12^{3} \times 24$ & 0.14077 & 0.14077 & $0.8153_{-38}^{+50}$ & $4-12$ & 39.6 & 40 & 0.488 \\
& & & & & & & & \\
1.0 & $16^{3} \times 32$ & 0.14663 & 0.14663 & $0.8950_{-21}^{+41}$ & $4-16$ & 24.2 & 31 & 0.802 \\
1.0 & $16^{3} \times 32$ & 0.14948 & 0.14663 & $0.8325_{-23}^{+51}$ & $4-16$ & 20.9 & 31 & 0.913 \\
1.0 & $16^{3} \times 32$ & 0.14948 & 0.14948 & $0.7680_{-35}^{+61}$ & $4-16$ & 22.0 & 31 & 0.882 \\
& & & & & & & & \\
1.57 & $16^{3} \times 32$ & 0.13843 & 0.13843 & $0.9357_{-28}^{+52}$ & $4-16$ & 21.7 & 31 & 0.893 \\
1.57 & $16^{3} \times 32$ & 0.14077 & 0.13843 & $0.8743_{-38}^{+64}$ & $4-16$ & 22.7 & 31 & 0.861 \\
1.57 & $16^{3} \times 32$ & 0.14077 & 0.14077 & $0.8093_{-50}^{+91}$ & $4-16$ & 24.0 & 31 & 0.811 \\
\hline \hline
\end{tabular}


TABLE IV. Nucleon masses at both volumes and values of $c$.

\begin{tabular}{cccccccc}
\hline \hline$c$ & $N_{s}^{3} \times N_{t}$ & $\kappa_{1}$ & $a m_{N}$ & Fit range & $\chi^{2}$ & $N_{\mathrm{DF}}$ & $Q$ \\
\hline 1.57 & $12^{3} \times 24$ & 0.13843 & $1.4147_{-53}^{+62}$ & $2-11$ & 2.0 & 14 & 0.999 \\
1.57 & $12^{3} \times 24$ & 0.14077 & $1.1741_{-94}^{+106}$ & $2-11$ & 6.4 & 14 & 0.955 \\
& & & & & & & \\
1.0 & $16^{3} \times 32$ & 0.14663 & $1.3948_{-71}^{+96}$ & $2-15$ & 20.3 & 22 & 0.564 \\
1.0 & $16^{3} \times 32$ & 0.14948 & $1.1667_{-84}^{+159}$ & $2-15$ & 25.4 & 22 & 0.279 \\
1.57 & $16^{3} \times 32$ & 0.13843 & $1.4231_{-79}^{+87}$ & $2-15$ & 17.6 & 22 & 0.728 \\
1.57 & $16^{3} \times 32$ & 0.14077 & $1.1853_{-116}^{+187}$ & $2-15$ & 23.1 & 22 & 0.397 \\
\hline \hline
\end{tabular}

is conservatively estimated to be the deviation of the quadratic fit from the original linear fit.

We note that the value for $\kappa_{\text {crit }}$ is always larger when the quadratic form is employed, regardless of the clover coefficient or lattice size used. Hence, results for other observables will always be quoted with an entirely positive or negative systematic error.

As can be seen from Table VI (including the GF11 [12] data for comparison), $\kappa_{\text {crit }}$ approaches $1 / 8$ as $c$ is increased from 0 to 1 and that $\widetilde{\kappa}_{\text {crit }}$ in the tadpole improved case is closer still.

\section{Meson masses}

In this section, the physical pseudoscalar and vector masses are evaluated by extrapolation and interpolation in the quark masses to the appropriate physical values. Certain input parameters are necessary to do this. In particular, for mesons containing up and down valence quarks (which are assumed to be degenerate in mass and will be referred to here as "normal"), one may use the experimental values for $M_{\pi}$ and $M_{\rho}$ (we apply a convention that experimentally determined masses are labeled with an " $M$," while those calculated on the lattice are labeled with an ' $m$ '). Effectively, one of these sets the quark mass while the other sets the lattice spacing.

The vector mass extrapolation has the following form:

$$
a m_{V}=a m_{\rho}^{\mathrm{crit}}+c_{V}\left(a m_{P}\right)^{2}+O\left(\left(a m_{P}\right)^{3}\right),
$$

where logarithmic terms due to the quenched approximation have been discarded. The constant term $a m_{\rho}^{\text {crit }}$ corresponds to the vector mass in the chiral limit. Following the procedure outlined by the GF11 collaboration, values of $a m_{\pi}$ and $a m_{\rho}$ are determined using the physical ratio

TABLE V. $\Delta$ masses at both volumes and values of $c$.

\begin{tabular}{cccccccc}
\hline \hline$c$ & $N_{s}^{3} \times N_{t}$ & $\kappa_{1}$ & $a m_{\Delta}$ & Fit range & $\chi^{2}$ & $N_{\mathrm{DF}}$ & $Q$ \\
\hline 1.57 & $12^{3} \times 24$ & 0.13843 & $1.5447_{-68}^{+87}$ & $5-11$ & 8.3 & 8 & 0.409 \\
1.57 & $12^{3} \times 24$ & 0.14077 & $1.3564_{-127}^{+156}$ & $5-11$ & 3.2 & 8 & 0.922 \\
& & & & & & & \\
1.0 & $16^{3} \times 32$ & 0.14663 & $1.4834_{-57}^{+112}$ & $5-15$ & 11.0 & 16 & 0.808 \\
1.0 & $16^{3} \times 32$ & 0.14948 & $1.2812_{-92}^{+180}$ & $5-15$ & 11.4 & 16 & 0.782 \\
1.57 & $16^{3} \times 32$ & 0.13843 & $1.5251_{-80}^{+125}$ & $5-15$ & 10.4 & 16 & 0.845 \\
1.57 & $16^{3} \times 32$ & 0.14077 & $1.3167_{-143}^{+207}$ & $5-15$ & 16.1 & 16 & 0.445 \\
\hline \hline
\end{tabular}

TABLE VI. Results for $\kappa_{\text {crit }}\left(\widetilde{\kappa}_{\text {crit }}\right.$ in the tadpole improved case), including the GF11 data at this $\beta$ for comparison. In the case of the UKQCD data, the first error quoted is statistical and the second is the systematic shift due to the fit to a quadratic form. The value of $\chi^{2} / N_{\mathrm{DF}}$ quoted is for the linear fit.

\begin{tabular}{lllc}
\hline \hline$N_{s}^{3} \times N_{t}$ & \multicolumn{1}{c}{$c$} & \multicolumn{1}{c}{$\kappa_{\text {crit }}$} & $\chi^{2} / N_{\mathrm{DF}}$ \\
\hline $12^{3} \times 24$ & 1.57 & $0.123480_{-15}^{+15}+238$ & 23.0 \\
& & & \\
$16^{3} \times 32$ & $0.0(\mathrm{GF} 11)$ & $0.169405 \pm 52$ (stat) & \\
$16^{3} \times 32$ & 1.0 & $0.153184_{-38}^{+37}+268$ & 4.5 \\
$16^{3} \times 32$ & 1.57 & $0.123466_{-26}^{+27}+176$ & 5.5 \\
\hline \hline
\end{tabular}

$$
\frac{a m_{\pi}}{a m_{\rho}}=\frac{M_{\pi}}{M_{\rho}}=0.1792 .
$$

Once again, the systematic error due to higher-order corrections is estimated by quadratically fitting all three masses and performing a linear fit in the two lightest masses. The deviation due to the quadratic fit was again found to be consistently larger. An example of this is shown in Fig. 6. The resulting values for $a m_{\rho}$ (including the GF11 [12] data) are quoted in Table VII. Having used the ratio of Eq. (15) to fix the normal quark mass, the scale can be determined using either $m_{\pi}$ or $m_{\rho}$.

It is useful to compare $m_{\rho}$ with the lattice measurement of a gluonic quantity, where discretization errors are $O\left(a^{2}\right)$ and, hence, can be expected to be smaller. We choose Sommer's force parameter, $r_{0}$ [24]. We can extrapolate the GF11 values for $m_{\rho} r_{0}$ versus $a r_{0}^{-1}$ to the continuum limit which yields

$$
\left.m_{\rho} r_{0}\right|_{a=0} ^{\text {quenched }}=2.03 \pm 0.07
$$

This includes a correction which the GF11 Collaboration have used to eliminate finite volume effects, which rounds

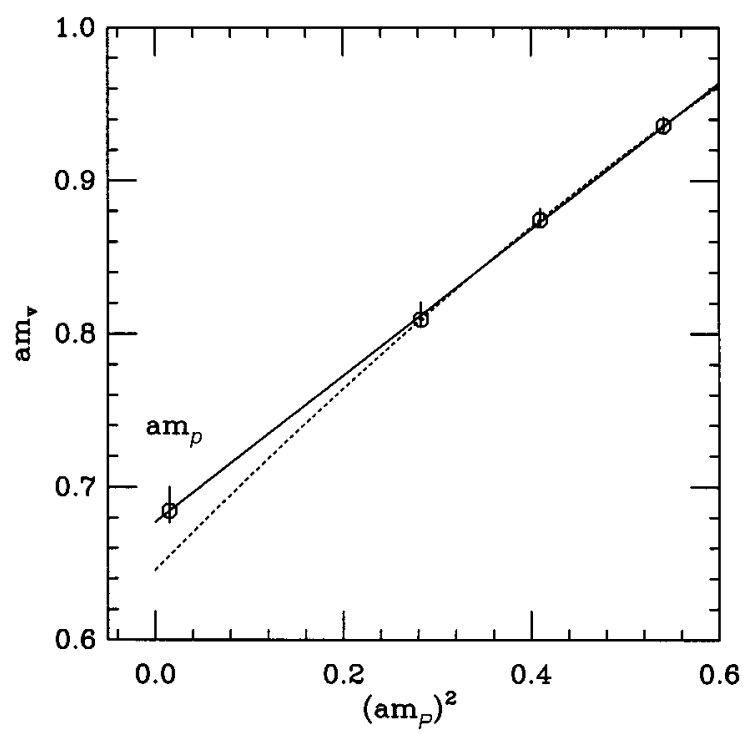

FIG. 6. $a m_{V}$ as a function of $\left(a m_{P}\right)^{2}, N_{s}^{2} \times N_{t}=16^{3} \times 32$, $c=1.57$. The solid line indicates the central value for the linear fit to all three masses. The dashed line is the quadratic fit. The calculated value for $a m_{\rho}$ for the linear fit is also quoted. 
TABLE VII. Chirally extrapolated results for the vector and pseudoscalar, at the zero and normal quark mass limits. The GF11 data at this $\beta$ is included for comparison. Another value for $a m_{\rho}$ was also computed by GF11 using a different smearing radius which is approximately 1-2 standard deviations smaller than the one quoted here. In the case of the UKQCD data, the first error quoted is statistical and the second is the systematic shift due to using quadratic chiral extrapolations. The value of $\chi^{2} / N_{\mathrm{DF}}$ quoted is for the linear fit.

\begin{tabular}{llcccc}
\hline \hline$N_{s}^{3} \times N_{t}$ & \multicolumn{1}{c}{$c$} & \multicolumn{1}{c}{$a m_{\pi}$} & \multicolumn{1}{c}{$a m_{\rho}^{\text {crit }}$} & \multicolumn{1}{c}{$a m_{\rho}$} & $\chi^{2} / N_{\mathrm{DF}}$ \\
\hline $12^{3} \times 24$ & 1.57 & $0.1250_{-13}^{+14}-59$ & $0.6897_{-75}^{+77}-349$ & $0.6969_{-74}^{+77}-327$ & 6.7 \\
$16^{3} \times 32$ & $0.0(\mathrm{GF} 11)$ & & & $0.5676 \pm 79$ (stat) & \\
$16^{3} \times 32$ & 1.0 & $0.1113_{-15}^{+15}-56$ & $0.6143_{-86}^{+85}-325$ & $0.6208_{-86}^{+84}-309$ & 2.4 \\
$16^{3} \times 32$ & 1.57 & $0.1228_{-19}^{+20}-60$ & $0.6778_{-106}^{+113}-357$ & $0.6850_{-106}^{+113}-336$ & 2.1 \\
\hline \hline
\end{tabular}

the result down by approximately $4 \%$. Assuming that $r_{0}$ and the string tension, $\sqrt{K}$ are related by $r_{0} \sqrt{K}=1.18$ and interpolating the available string tension data from $\beta=5.7-6.5$, one finds $r_{0} / a$ at $\beta=5.7$ to be 2.94. One can then compare our data for $m_{\rho} r_{0}$ at $\beta=5.7$ as a function of $c$ with the continuum limit from GF11. These results are plotted as a function of $c$ in Fig. 7, noting that there are significant discretization effects in the force parameter at $\beta=5.7$ which have not been taken into account. There is a clear trend toward the continuum limit as the clover coefficient is increased to its tadpole improved value.

The determination of meson masses containing strange valence quarks requires as input the experimental mass of a strange meson, for example, $M_{K}$. With this mass as input, one can determine $a m_{K}$ by requiring:

$$
\frac{a m_{K}}{a m_{\rho}}=\frac{M_{K}}{M_{\rho}}=0.643 .
$$

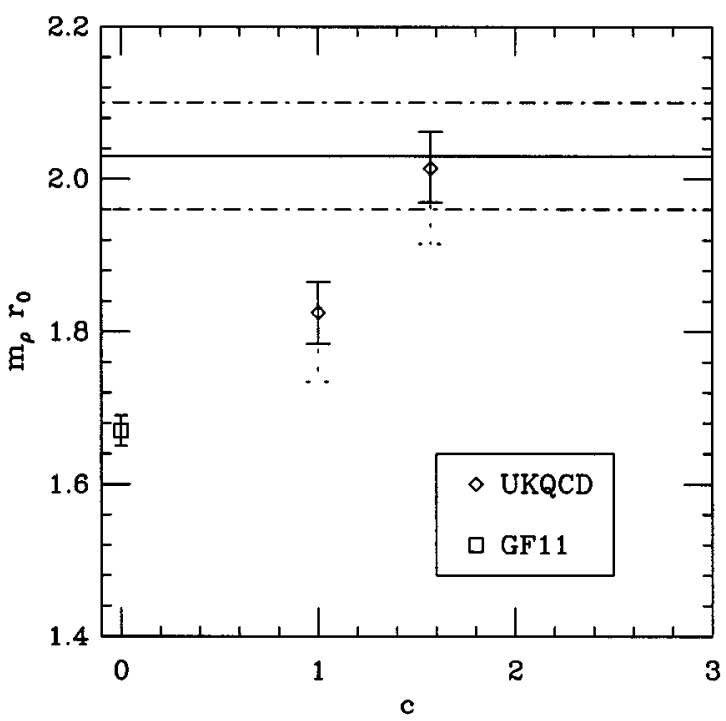

FIG. 7. $m_{\rho} r_{0}$ versus $c$. Statistical errors on the data points are marked with solid lines. Systematic errors due to the quadratic chiral extrapolation are marked, on the data points, with dashed lines. The horizontal lines indicate the continuum limit from the GF11 data (a finite-volume correction has been included), along with the statistical error of the fit to the continuum. Systematic effects due to discretization errors in $r_{0}$, which are $O\left(a^{2}\right)$ (necessary for the extrapolation to the continuum), have not been included.
From the condition of Eq. (17) and employing Eq. (14), one can then predict $a m_{K^{*}}$ fixed from $a m_{K}$, which we refer to as $a m_{K *}\left(a m_{K}\right)$. Our results for $a m_{K *}\left(a m_{K}\right)$ and the ratio $\left[a m_{K} *\left(a m_{K}\right)\right] / a m_{\rho}$ can be found in columns 3 and 4 of Table VIII. We note that the ratio $\left[a m_{K *}\left(a m_{K}\right)\right] / a m_{\rho}$ at $c=1$ is consistent to within 1 standard deviation with that at $c=1.57$ and that the central value lies several standard deviations below the experimental value. There are large systematic errors due to the chiral extrapolation at both values of the clover coefficient, however, this error is smaller than the difference between our results and experimental data. The discrepancy in this ratio has also been noted at $\beta=6.0$, with $c=0$ by Bhattacharya et al. [22].

The choice of strange meson is not unique. Instead, one could have fixed $a m_{K^{*}}$ from

$$
\frac{a m_{K^{*}}}{a m_{\rho}}=\frac{M_{K^{*}}}{M_{\rho}}=1.160,
$$

and through Eq. (14) one can then predict $a m_{K}$ fixed from $a m_{K^{*}}$, which we refer to as $a m_{K}\left(a m_{K^{*}}\right)$. Our results at both clover coefficients for this mass and the ratio $\left[a m_{K}\left(a m_{K^{*}}\right)\right] / a m_{\rho}$ are listed in columns 5 and 6 of Table VIII. We note that the ratio $\left[\operatorname{am}_{K}\left(a m_{K^{*}}\right)\right] / a m_{\rho}$ is also constant to within one standard deviation as $c$ is changed from 1 to 1.57 and that the central value lies several standard deviations above the experimental value. However, in this case, the systematic errors due to the chiral extrapolation at both values of the clover coefficient are so large that we cannot demonstrate that these ratios are inconsistent with experiment.

The mass $a m_{\phi}$ of the pure valence $\overline{s s}$ vector state can be determined similarly, but a valence $\overline{s s}$ pseudoscalar, $\eta_{s}$, is not observed. However, using an estimate of $M_{\eta_{s}}$ by Lipps et al. [25], we can estimate the ratio of these masses:

$$
\frac{M_{\phi}}{{ }^{\prime} M_{\eta_{s}}, "} \approx \frac{a m_{V}(\overline{s s})}{a m_{P}(\overline{s s})}=1.5 .
$$

It is therefore possible to determine $\operatorname{am}_{V}(\overline{s s})$, from Eq. (14) and Eq. (19) without extrapolating to the chiral limit, which we have seen previously, has large systematic errors. The resulting masses are shown in Table IX.

Using the data from the GF11 Collaboration, it is possible to calculate $\operatorname{am}_{V}(\overline{s s})$ for $c=0$ for $\beta=5.7$ and the other gauge couplings. Assuming a linear behavior with respect to 
TABLE VIII. Results for the strange mesons at both volumes and $c$, using $M_{K}$ (columns 3 and 4) and $M_{K^{*}}$ (columns 5 and 6) to fix the strange quark mass. The first error quoted is statistical and the second is the systematic shift due to the use of a quadratic chiral extrapolation.

\begin{tabular}{cccccc}
\hline \hline$N_{s}^{3} \times N_{t}$ & $c$ & $a m_{K^{*}}\left(a m_{K}\right)$ & {$\left[a m_{K^{*}}\left(a m_{K}\right)\right] / a m_{\rho}$} & $a m_{K}\left(a m_{K^{*}}\right)$ & {$\left[a m_{K}\left(a m_{K^{*}}\right)\right] / a m_{\rho}$} \\
\hline $12^{3} \times 24$ & 1.57 & $0.782 \pm 7-18$ & $1.122 \pm 2+28$ & $0.505 \pm 9-65$ & $0.724 \pm 6-94$ \\
$16^{3} \times 32$ & 1.0 & $0.698 \pm 9-21$ & $1.124 \pm 2+24$ & $0.447_{-9}^{+8}-53$ & $0.721 \pm 6-85$ \\
$16^{3} \times 32$ & 1.57 & $0.771_{-10}^{+11}-21$ & $1.125 \pm 3+28$ & $0.491_{-12}^{+13}-60$ & $0.717 \pm 8-88$ \\
\hline \hline
\end{tabular}

the lattice spacing, the continuum limit of $m_{V}(\overline{s s}) r_{0}$ using the GF11 data has been evaluated. It should be noted, however, that the linear extrapolation in the lattice spacing for the GF11 data is very poor, having a $\chi^{2} / N_{\mathrm{DF}}$ of approximately 13 , even though the fit is uncorrelated. It is likely, therefore, that the continuum limit for $m_{V}(\overline{s s}) r_{0}$ has a large systematic error due to this fit. There is also a correction to infinite volume which shifts the value downwards. The behavior of $m_{V}(\overline{s s}) r_{0}$ with respect to $c$ at $\beta=5.7$ is shown in Fig. 8. The absence of the systematic error due to the chiral extrapolation demonstrates the effect of the clover coefficient more clearly than from $m_{\rho} r_{0}$. Again, we find there is a clear trend toward the continuum limit as the clover coefficient is increased to its tadpole improved value.

\section{Mass splittings}

Heavy quark effective theory (HQET) predicts that for heavy-light mesons, the vector-pseudoscalar mass splitting, $\Delta_{V-P}=m_{V}^{2}-m_{P}^{2}$ is constant. This is borne out by experiment, with $M_{D *}^{2}-M_{D}^{2} \approx 0.53 \quad \mathrm{GeV}^{2}$ and $M_{B *}^{2}-M_{B}^{2}$ $\approx 0.49 \mathrm{GeV}^{2}$. A somewhat unexpected experimental result is that this trend is continued into the light quark regime, where the hyperfine splitting, $\Delta_{V-P}$, remains approximately constant at $0.55 \mathrm{GeV}^{2}$.

Quenched lattice simulations fail noticeably to reproduce this behavior. HQET predicts that $\Delta_{V-P}$ is proportional to $\left\langle\bar{h} \sigma_{\mu \nu} F^{\mu \nu} h\right\rangle$, where $h$ is the heavy quark field. As the clover term is of this form, naively one would then expect that increasing the size of clover coefficient would reduce this discrepancy at least for heavy-light systems. Tentative comparisons with the $c=0$ and $c=1$ actions at $\beta=6.2$ with low statistics indicated that the fall off in the splitting had decreased [3].

In Fig. 9 the splittings from the three different values of the clover coefficient are compared. The scale for each action is chosen from $M_{K^{*}}$. The slope $\partial\left(a^{2} \Delta_{V-P}\right) / \partial\left(a m_{P}\right)^{2}$ is unaffected by this choice. While there is a noticeable change in the slope on going from $c=0$ to $c=1$, the slopes at $c=1$ and

TABLE IX. Masses for the valence $\overline{s s}$ states, defined from fixing the ratio $a m_{V}(\overline{s s}) / a m_{P}(\overline{s s})$ to 1.5 .

\begin{tabular}{ccccc}
\hline \hline$N_{s}^{3} \times N_{t}$ & $c$ & $a m_{V}(\overline{s s})$ & $a m_{P}(\overline{s s})$ & $\chi^{2} / N_{\mathrm{DF}}$ \\
\hline $12^{3} \times 24$ & 1.57 & $0.831 \pm 6$ & $0.554 \pm 4$ & 6.7 \\
$16^{3} \times 32$ & 1.0 & $0.742_{-8}^{+7}$ & $0.495 \pm 5$ & 2.4 \\
$16^{3} \times 32$ & 1.57 & $0.821_{-9}^{+10}$ & $0.547_{-6}^{+7}$ & 2.1 \\
\hline \hline
\end{tabular}

$c=1.57$ are consistent with each other. The remaining discrepancy is presumably due to the error of the quenched approximation.

\section{The J parameter}

As noted previously, it is useful to be able to compare lattice spectrum results with existing experimental data without an extrapolation to the chiral limit. The parameter $J$, defined as [13]

$$
J \equiv m_{K^{*}} \frac{d m_{V}}{d m_{P}^{2}}, \quad \frac{m_{K^{*}}}{m_{K}}=1.8 .
$$

allows such a comparison. Existing quenched Wilson-like fermion actions yield values around $J=0.37$ whereas an estimate of $J$ using experimental data yields $J=0.48(2)$. In Fig. 10, $J$ versus $c$ (including the calculated value of $J$ at two volumes from the GF11 Collaboration) is plotted. We find

$$
\begin{gathered}
J\left(\beta=5.7, c=1,16^{3} \times 32\right)=0.361 \pm 7, \\
J\left(\beta=5.7, c=1.57,16^{3} \times 32\right)=0.366 \pm 10 .
\end{gathered}
$$

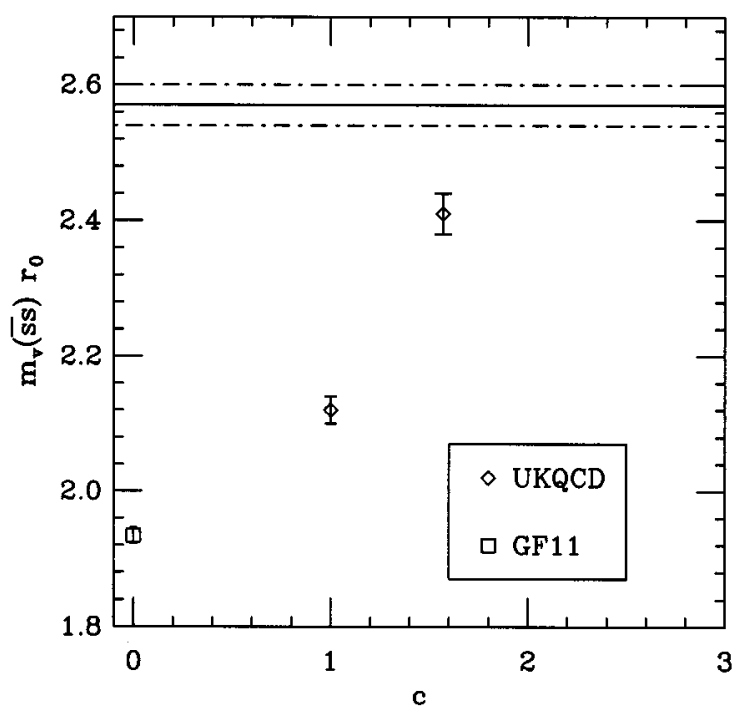

FIG. 8. $m_{V}(\overline{s s}) r_{0}$ versus $c$. The horizontal line indicates the continuum limit from the GF11 data (a finite-volume correction has been included). Systematic effects due to discretization errors in $r_{0}$, which are $O\left(a^{2}\right)$ (necessary for the extrapolation to the continuum), have not been included. 


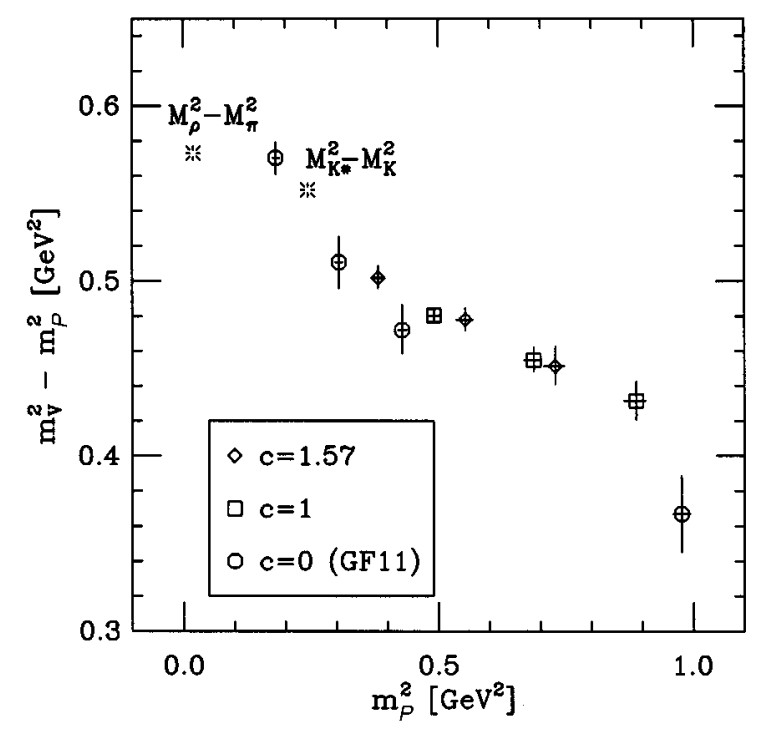

FIG. 9. The hyperfine splitting $m_{V}^{2}-m_{P}^{2}$ versus $m_{P}^{2}$ for all the three values of $c$ at $\beta=5.7,16^{3} \times 32$.

The values of $J$ from Eq. (21) and Eq. (24) below, for both nonzero values of $c$ and both volumes, agree with the world average of the quenched data, and disagree with the experimental estimate. It should be noted that $J$ is trivially related to the slope $\partial\left(a^{2} \Delta_{V-P}\right) / \partial\left(a m_{P}\right)^{2}$ outlined in the previous section. We, therefore, expect that the prescription that solves the anomalous behavior of $\Delta_{V-P}$ will also solve the disagreement in $J$.

\section{Baryons}

We extrapolate the nucleon mass to the normal-quark limit assuming a linear dependence on the quark mass:

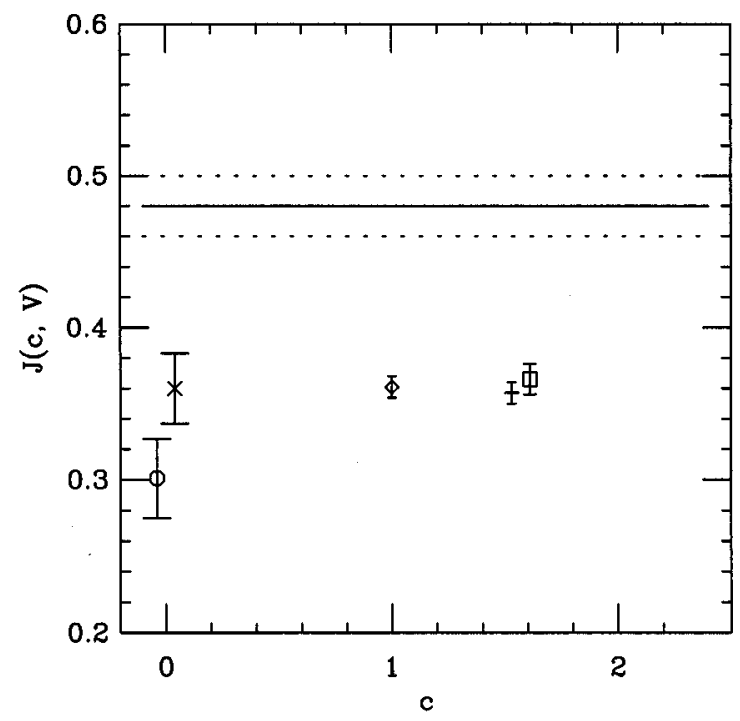

FIG. 10. The parameter $J$ against $c$ for all values of $c$ and volumes. The horizontal line indicates the "experimental value"; GF11, $c=0,16^{3} \times 32(\bigcirc)$; GF11, $c=0,24^{3} \times 32(\times)$; UKQCD, $c=1.0,16^{3} \times 32,(\diamond)$; UKQCD, $c=1.57,16^{3} \times 32,(\square)$; UKQCD, $c=1.57,12^{3} \times 24(+)$.
TABLE X. Chirally extrapolated results for the nucleon, at the zero and normal quark mass limit. The $\chi^{2} / N_{\mathrm{DF}}$ is not quoted as the number of degrees of freedom is equal to the number of data points.

\begin{tabular}{llll}
\hline \hline$N_{s}^{3} \times N_{t}$ & $c$ & $a m_{N}^{\text {crit }}$ & $a m_{N}\left(a m_{\pi}\right)$ \\
\hline $12^{3} \times 24$ & 1.57 & $0.916_{-15}^{+16}$ & $0.931_{-15}^{+15}$ \\
$16^{3} \times 32$ & 1.0 & $0.891_{-24}^{+23}$ & $0.902_{-23}^{+23}$ \\
$16^{3} \times 32$ & 1.57 & $0.931_{-28}^{+30}$ & $0.945_{-28}^{+29}$ \\
\hline \hline
\end{tabular}

$$
a m_{N}=a m_{N}^{\text {crit }}+c_{N}\left(a m_{P}\right)^{2}+O\left(\left(a m_{P}\right)^{2}\right) .
$$

We extrapolate the $\Delta$ mass likewise. The final results for the nucleon and $\Delta$ are quoted in Table X and Table XI, respectively.

From the combined results for the pseudoscalar, vector, and nucleon masses, we show the "Edinburgh" plot in Fig. 11. One finds a statistically significant difference between the ratios at each value of $c$. As $c$ is increased, the trend of the data is towards the phenomenological curve of Ono [26]. Furthermore, the ratio $m_{N} / m_{\rho}$ approaches the experimental value $M_{N} / M_{\rho}$, but even at $c=1.57$ is still approximately $13 \%$ too large.

\section{B. Finite volume effects}

The masses obtained for the $12^{3} \times 24$ lattice are listed in Table II to Table V. As stated previously, it proved to be somewhat more difficult to extract reliable masses for this volume. As before, $\widetilde{\kappa}_{\text {crit }}$ is evaluated with a statistical and systematic error to be

$$
\widetilde{\kappa}_{\text {crit }}\left(\beta=5.7, c=1.57,12^{3} \times 24\right)=0.12348_{-2}^{+2}+24,
$$

which agrees with the result from the larger volume and has a similarly sized systematic error. Likewise, as shown in Fig. 12, the hyperfine splittings are consistent to within 1 standard deviation. The chirally extrapolated and strange meson masses are determined as in Sec. III A 2 and the results listed in Table VII and Table VIII. Once again, the results are consistent to within one standard deviation with those on the larger volume. Similarly, the parameter $J$ is determined to be

$$
J\left(\beta=5.7, c=1.57,12^{3} \times 24\right)=0.357 \pm 7,
$$

which is consistent with the larger volume.

TABLE XI. Chirally extrapolated $\Delta$ masses, at the zero and normal quark mass limit. The $\chi^{2} / N_{\mathrm{DF}}$ is not quoted as the number of degrees of freedom is equal to the number of data points.

\begin{tabular}{llll}
\hline \hline$N_{s}^{3} \times N_{t}$ & $c$ & $a m_{\Delta}^{\text {crit }}$ & $a m_{\Delta}\left(a m_{\pi}\right)$ \\
\hline $12^{3} \times 24$ & 1.57 & $1.156_{-24}^{+23}$ & $1.167_{-23}^{+22}$ \\
$16^{3} \times 32$ & 1.0 & $1.036_{-24}^{+25}$ & $1.047_{-24}^{+25}$ \\
$16^{3} \times 32$ & 1.57 & $1.091_{-30}^{+30}$ & $1.103_{-30}^{+29}$ \\
\hline \hline
\end{tabular}




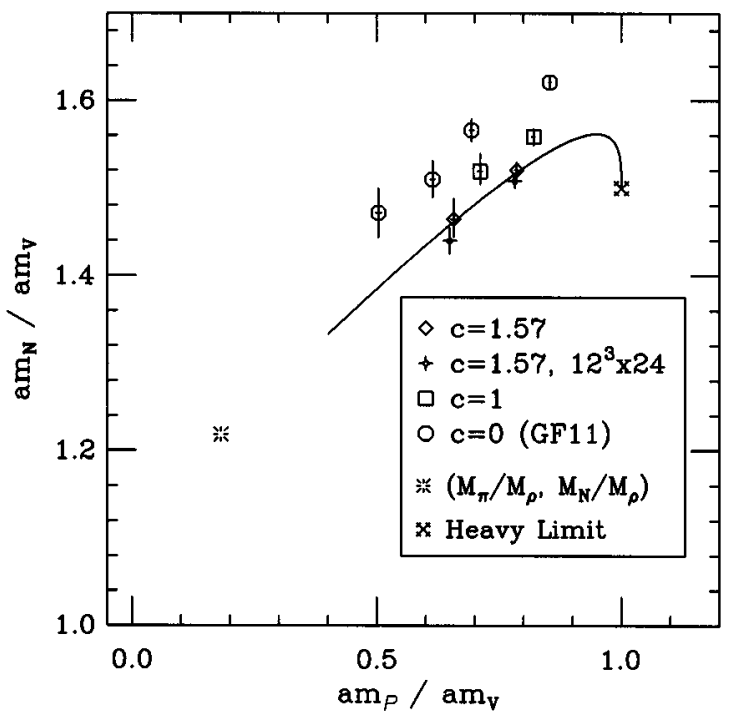

FIG. 11. The Edinburgh plot for all three values of $c$.

Both baryons are more strongly affected by the size of the lattice. The nucleon masses at both $\kappa$ 's are approximately two standard deviations smaller than in the larger volume. This is somewhat unexpected as other studies in quenched QCD using Wilson-like fermions indicate that the nucleon mass falls with increasing size over a similar range of volumes [using $a m_{\rho}$ to determine the lattice spacing, we see that our volumes vary from $(2 \mathrm{fm})^{3}$ to $(2.6 \mathrm{fm})^{3}$ approximately]. We note that the $Q$ values for the fit to the nucleon masses on the $12^{3} \times 24$ lattice are very close to 1 , which may indicate that the statistical errors are underestimated. We find the extrapolated value

$$
\operatorname{am}_{N}\left(\beta=5.7, c=1.57,12^{3} \times 24\right)=0.931_{-15}^{+15},
$$

which is also two standard deviations smaller than in the larger volume. The $\Delta$ masses at both $\kappa$ 's lie approximately two standard deviations above the values on the larger lattice, and

$$
\operatorname{am}_{\Delta}\left(\beta=5.7, c=1.57,12^{3} \times 24\right)=1.167_{-23}^{+22} .
$$

\section{CONCLUSIONS}

In this paper, we have examined the effect, at $\beta=5.7$, of changing the clover coefficient and volume on the quenched light-hadron spectrum computed using the SW fermion action. As the clover coefficient is increased, there is better agreement between the perturbative (tree-level) and nonperturbative calculation of $\kappa_{\text {crit }}$. When the clover coefficient is changed from $c=0$ (the Wilson action) to $c=1$ (the SW action) and $c=1.57$ (the tadpole-improved SW action) there is a significant difference in the masses of the chirally extrapolated and strange pseudoscalar and vector mesons, in the nucleon and $\Delta$ masses and in the Edinburgh plot.

Interestingly, a number of other quantities, for example $m_{V}^{2}-m_{P}^{2}, J$ and the ratios $a m_{K} / a m_{\rho}$ and $a m_{K^{*}} / a m_{\rho}$ do not appear to change significantly as $c$ is changed from 1.0 to

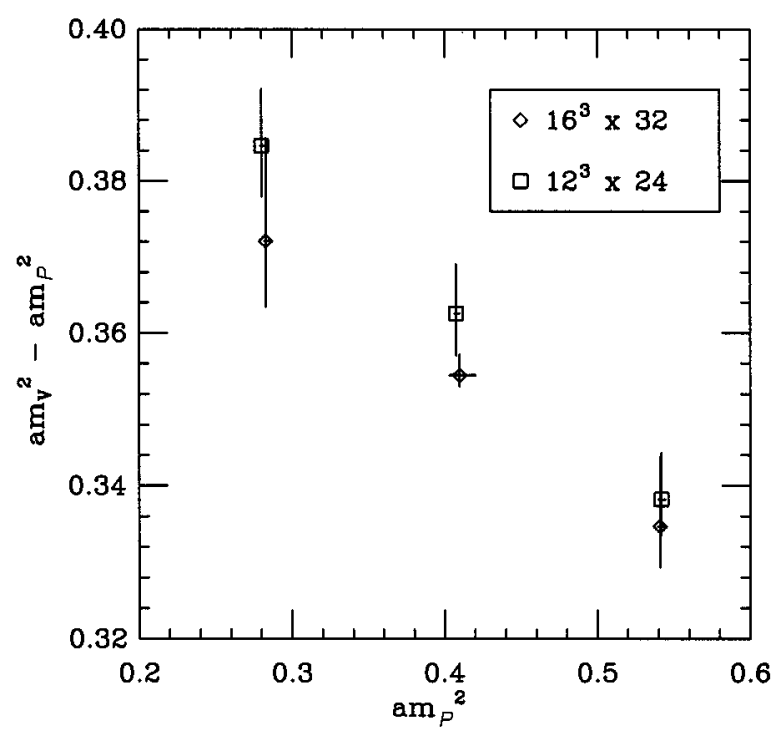

FIG. 12. $\left(a m_{V}\right)^{2}-\left(a m_{P}\right)^{2}$ plotted against $\left(a m_{P}\right)^{2}$ for $c=1.57$, $N_{s}^{2} \times N_{t}=16^{3} \times 32$ and $12^{3} \times 24$.

1.57. As the finite volume effects appear to be under control, and these observables have been chosen to avoid the systematic errors due to the chiral extrapolation, the possible remaining systematic errors are the effect of quenching the gauge configurations and a possibly large deviation of the mean field estimate of the clover coefficient from $c_{\text {nonpert }}$. It would, therefore, be very interesting then to examine the behavior of these quantities in any future studies in full QCD under changes in the value of the clover coefficient.

In changing the volume from approximately $(2 \mathrm{fm})^{3}$ to $(2.6 \mathrm{fm})^{3}$, the mesonic observables are consistent to within one standard deviation. Baryon masses are consistent to within two standard deviations. Unfortunately, with this data, one cannot differentiate between different Ansätze used for describing the volume behavior of masses $[27,28]$.

The Alpha Collaboration $[5,29]$ has calculated the clover coefficient nonperturbatively for $6.0 \leqslant \beta \leqslant 6.8$. In general, the coefficients obtained through this approach are significantly larger than those obtained via tadpole improvement, although the coefficients converge as $\beta$ is increased. Our data appears to suggest that $c_{\text {nonpert }}$ could at $\beta=5.7$ be somewhat larger than the tadpole improved value.

Currently, we are carrying out an analysis of the quenched light hadron mass spectrum at $\beta=6.0$ and $\beta=6.2$ using the tadpole improved SW action [30]. This will directly explore whether better scaling is achieved using $c=1 / u_{0}^{3}$ than with $c=1$.

\section{ACKNOWLEDGMENTS}

H.P.S. would like to thank the staff at Brookhaven National Laboratory for their kind hospitality during his stay there. D.G.R. acknowledges financial support from PPARC and the support of Argonne National Laboratory during the completion of this work. The UKQCD Collaboration wish to express their thanks to the Edinburgh Parallel Computing Centre for their support and maintenance of the Meiko Computing Surfaces. 
[1] K. Symanzik, Mathematical Problems in Theoretical Physics, edited by R. Schrader et al., Lecture Notes in Physics Vol. 153 (Springer Verlag, Berlin, 1982); K. Symanzik, Proceedings of the Trieste Workshop on Nonperturbative Field Theory and QCD (World Scientific, Singapore, 1982), p. 61.

[2] B. Sheikholeslami and R. Wohlert, Nucl. Phys. B259, 572 (1985).

[3] UKQCD Collaboration, C. R. Allton et al., Nucl. Phys. B407, 331 (1993).

[4] M. Lüscher and P. Weisz, Commun. Math. Phys. 97, 59 (1985).

[5] Alpha Collaboration, K. Jansen et al., Phys. Lett. B 372, 275 (1996).

[6] S. Collins et al., in Lattice '95, Proceedings of the International Symposium, Melbourne, Australia, edited by T. D. Kiev et al. [Nucl. Phys. B (Proc. Suppl.) 47, 366 (1996)]; Report No. hep-lat/9509043 (unpublished).

[7] M. Alford et al., in Lattice '95 [6], p. 370, Report No. hep-lat/ 9509087 (unpublished).

[8] C. Morningstar and M. Peardon, Nucl. Phys. B (Proc. Suppl.) 47, 258 (1996); Report No. hep-lat/9509069 (unpublished).

[9] H. R. Fiebig and R. M. Woloshyn, Report No. TRI-PP-96-1, hep-lat/9603001, 1996 (unpublished).

[10] P. Hasenfratz and F. Niedermayer, Nucl. Phys. B414, 785 (1994).

[11] G. P. Lepage and P. B. Mackenzie, Phys. Rev. D 48, 2250 (1993).

[12] GF11 Collaboration, F. Butler et al., Nucl. Phys. B430, 179 (1990).
[13] UKQCD Collaboration, P. Lacock and C. Michael, Phys. Rev. D 52, 5213 (1995).

[14] M. Creutz, Phys. Rev. D 36, 515 (1987); F. R. Brown and T. J. Woch, Phys. Rev. Lett. 58, 2394 (1987).

[15] N. Cabibbo and E. Marinari, Phys. Lett. 119B, 387 (1982).

[16] UKQCD Collaboration, C. R. Allton et al., Phys. Rev. D 47, 5128 (1993).

[17] UKQCD Collaboration, C. R. Allton et al., Phys. Rev. D 49, 474 (1994).

[18] W. H. Press et al., Numerical Recipies in C, 2nd ed. (Cambridge University Press, Cambridge, England, 1992).

[19] D. W. Marquardt, J. Soc. Ind. Appl. Math. 11, 431 (1963).

[20] B. Efron, SIAM (Soc. Ind. Appl. Math.) Rev. 21, 460 (1979).

[21] S. Sharpe, Phys. Rev. D 41, 3233 (1990); 46, 3146 (1992).

[22] T. Bhattacharya et al., Phys. Rev. D 53, 6486 (1996).

[23] S. Collins et al., Nucl. Phys. B (Proc. Suppl.) 47, 451 (1996); Report No. hep-lat/9512027 (unpublished).

[24] R. Sommer, Nucl. Phys. B411, 839 (1994).

[25] H. Lipps et al., Phys. Lett. 126B, 250 (1983).

[26] S. Ono, Phys. Rev. D 17, 888 (1978).

[27] M. Lüscher, Commun. Math. Phys. 104, 177 (1986).

[28] M. Fukugita et al., Phys. Lett. B 294, 380 (1992).

[29] Alpha Collaboration, M. Lüscher et al., in Lattice '96, Proceedings of the International Symposium, St. Louis, Missouri [Nucl. Phys. B (Proc. Suppl.) (in press)], Report No. hep-lat/ 9608049 (unpublished).

[30] R. D. Kenway et al., in Lattice '96 [29]; UKQCD Collaboration (in preparation). 